\title{
In-situ measurements of surface (photo)voltage of roll-to-roll deposited thin film silicon solar cells
}

\author{
Bas B. Van Aken ${ }^{*}, 1$, Klaas J. Bakker ${ }^{1}$, Maurits C.R. Heijna ${ }^{1}$, Dennis Reid ${ }^{2}$, lain Baikie ${ }^{2}$ and Wim J. \\ Soppe ${ }^{1}$ \\ ${ }^{1}$ ECN Solar Energy, P.O. Box 1, NL-1755 ZG Petten, the Netherlands \\ ${ }^{2}$ KP Technology Ltd, Wick, United Kingdom, KW1 5EH
}

* Corresponding author: e-mail vanaken@ecn.nl, Phone: +31 22456 4905, Fax: +31 224568214

The Kelvin probe is a non-contact, non-destructive vibrating capacitor device that measures the work function difference between a conducting sample and a vibrating tip. This contribution focuses on inline monitoring of the surface (photo) voltage of deposited silicon layers. We apply a custom-built in-situ Kelvin probe, operated in a roll-to-roll PECVD system, located immediately after the plasma zones to enable direct feedback to the controlling system of the plasma deposition. The surface photovoltage of nip thin film Si solar cells increases with increasing Voc. The results imply that inline, contactless measurements of the open circuit voltage are possible and that thus monitoring the doped layer quality during roll-to-roll production is feasible.

\section{Introduction}

Roll-to-roll production of thin film Si solar cells has advantages over batch-type reactor systems, since it allows high-throughput fabrication and application of cheap foil substrates. The flexible substrate also enables broader application, e.g. in the built environment, which is the most important market in densely populated, developed countries [1, 2].

ECN is developing a pilot line for roll-to-roll production of high efficiency n-i-p solar cells based on amorphous (a-Si:H) and microcrystalline ( $\square \mathrm{c}-\mathrm{Si}: \mathrm{H})$ silicon thin films on steel foil, coated with an insulating barrier layer and sputtered back contact and reflector. The main purpose of the barrier layer is to enable monolithic series interconnection of cells after deposition of all layers [3].

In roll-to-roll production, inline quality assessment and control are of utmost importance. Being able to continuously check the quality of the deposited silicon, and instantaneously adjust the process when necessary, will greatly enhance the yield. Noncontact measurements, such as spectral ellipsometry $[4,5]$ and UV-VIS reflectometry [6, 7], are preferred in order not to damage the surface of the deposited layers. Kelvin probe measurements are a good addition to the optical measurements as they supply information on electrical properties without touching the deposited layers.

This contribution focuses on inline monitoring of the surface (photo)voltage of deposited silicon layers using an in-situ Kelvin probe in the so-called 'off-null' method [8]. We will present continuous measurements of roll-to-roll deposited Si layers with varying process parameters. Furthermore, we will also show the relation between the surface photovoltage and the open-circuit voltage of pin and nip thin film Si solar cells.

\section{Experimental set-up}

2.1 Kelvin probe principle The Kelvin probe is a non-contact, non-destructive vibrating capacitor device to measure the work function difference between a conducting sample and a vibrating probe. The probe-sample configuration can be regarded as a flatplate capacitor. Note that the work function is sensitive to the surface condition, e.g. native oxide layers or contamination, and also to external factors, such as illumination.

Fig. 1 shows the electron energy levels of probe and sample, both in open circuit situation and in contact. The work functions and Fermi levels of probe and sample are $\square_{\mathrm{p}}, \square_{\mathrm{s}}$, and $\square_{\mathrm{p}}, \square_{\mathrm{s}}$. We can determine the work function difference $\square_{p}-\square_{s}$, when the sample and probe are brought into contact as electrons will flow from the higher work function material to the lower work function material, until the charge redistribution equalises the Fermi levels of both materials. The resulting contact potential difference $V_{c}$ is equal to $\square_{p}-\square_{s}$. 


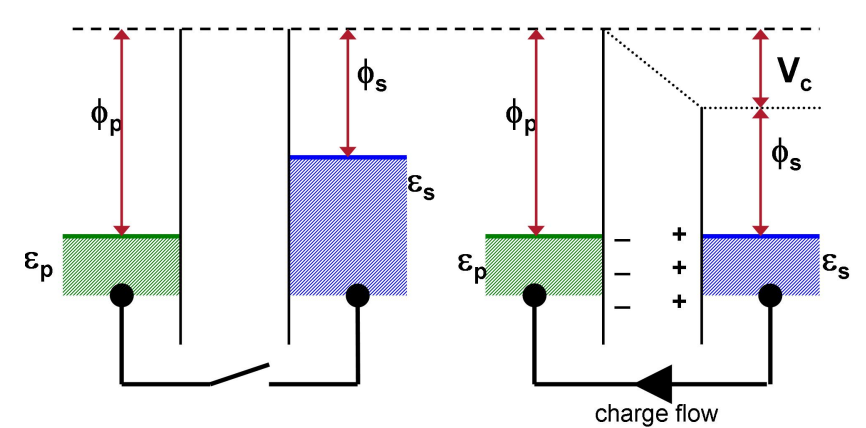

Figure 1 Electron energy level diagrams of sample and probe (a) without contact and (b) with external electrical contact.

Direct measurement of $V_{c}$, is almost impossible but a practical alternative is to find the bias voltage (usually called the backing voltage) at which the charge on the capacitor is null. This can be done by vibrating the probe, i.e. varying the probe-sample distance. This changes the capacitance of the device, leading to an oscillating current that can be measured with a lock-in amplifier. If the charge is zero then no current will be measured and the backing voltage equals the contact potential difference, $V_{b}=-V_{c}$.

However, determining this voltage exactly is still difficult as the Kelvin probe signal is very noise sensitive when the oscillating current approaches zero. We use an alternative method that measures the Kelvin probe signal at bias voltages far off-balance, the 'offnull' method [8], see Fig. 2. By determining the peakto-peak voltage $V_{\text {ptp }}$, which scales with the oscillating current, accurately for two or more bias voltages, we can interpolate the data to the contact potential, for which the $\mathrm{V}_{\mathrm{ptp}}=0$.

\subsection{In-situ installation}

A vacuum compatible Kelvin probe was custom built in collaboration with KP technology Ltd. It fits, including a $5-\mathrm{cm}$ vacuum translator, on a regular DN40CF flange. The integrated white light LED illumination unit allows measuring the surface voltage under illumination.

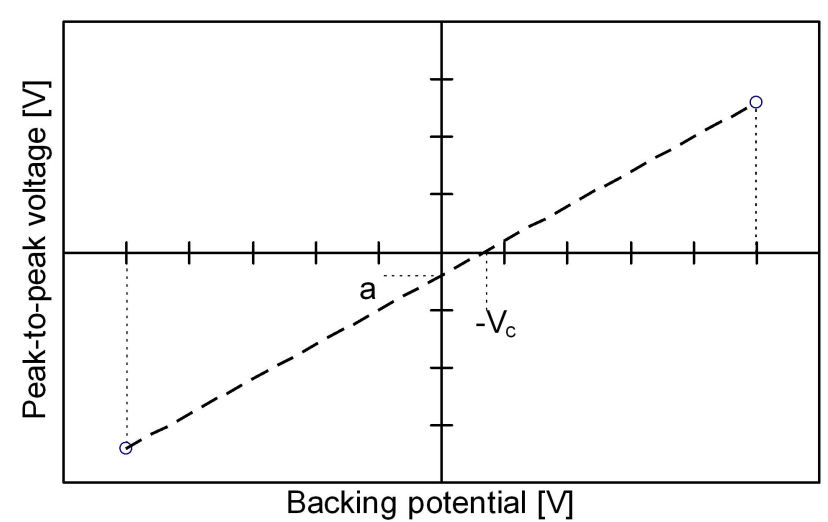

Figure 2 Sketch of the determination of $V_{c}$. In the off-null method $V_{\text {ptp }}=a+V_{b} \times G$, where the gradient $G$ is given by $\square \mathrm{V}_{\text {ptp }} / \square \mathrm{V}_{\mathrm{b}}$ and $\mathrm{V}_{\mathrm{c}}=-\mathrm{a} / \mathrm{G}$.

Besides the work function difference, also the gradient, i.e. $\square \mathrm{V}_{\mathrm{ptp}} / \square \mathrm{V}_{\mathrm{b}}$, is recorded, see Fig. 2. It follows from the theory that the slope is inversely proportional to the distance between the sample and the probe tip. However, changing the sample-probe distance also changes the surface voltage, as can be seen in Fig. 3. The KP Technology Ltd. system constantly monitors the fractional change in capacitance between the tip of the vibrating Kelvin probe and the foil. This parameter can be used to automatically keep the spacing between probe and foil fixed. This assures that changes in surface voltage are solely a consequence of changes in the sample properties and not of a deviation in the sample-probe distance.

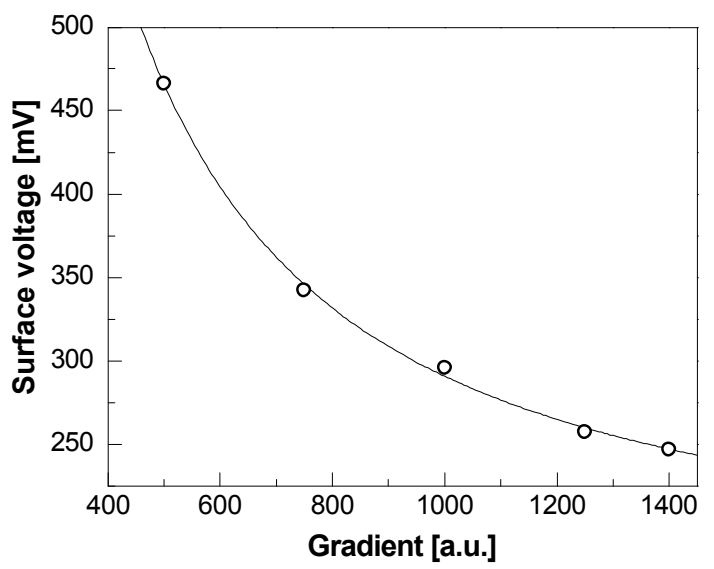

Figure 3 Surface voltage as a function of gradient measured on the steel foil. The gradient is varied by changing the sample-probe spacing. The solid line is a fit, assuming that the surface voltage is inversely proportional to the gradient. Measurements on different materials show the same curvature, differing by a vertical shift only. 


\section{Results and discussion}

First, we will show that although the Kelvin probe is sensitive both to sample (surface) properties and to the sample-probe distance, we can separate these two influences. We have stuck a steel substrate with an a-Si layer with tin plated cupper tape to the steel carrier foil, creating the sample stack shown in Fig. 4. We used the Kelvin probe under process conditions and moved the foil with $5 \mathrm{~cm} / \mathrm{min}$.

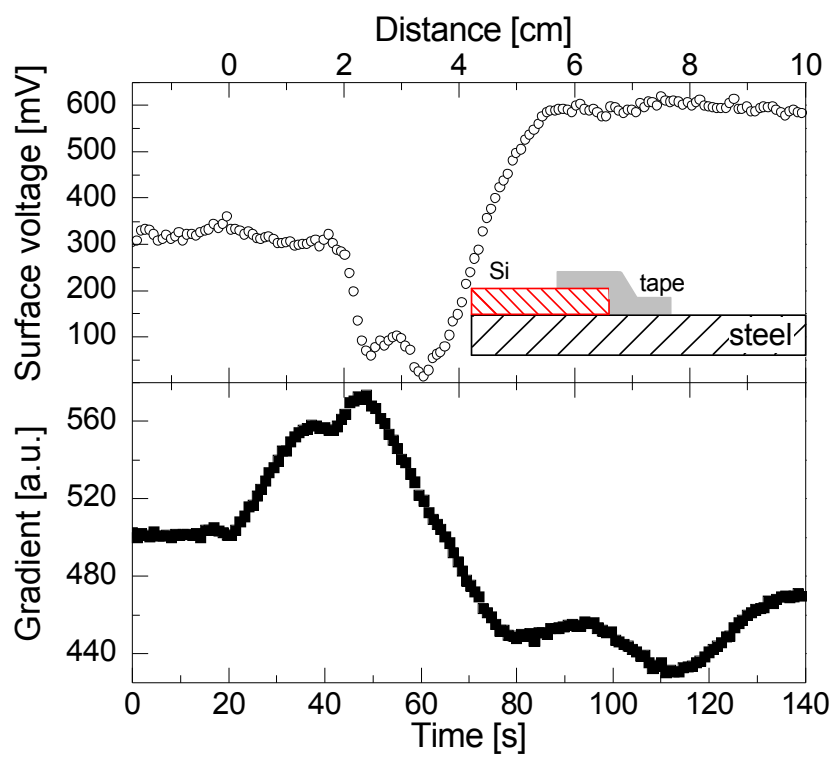

Figure 4 Surface voltage and gradient of the shown sample stack (width of tape on scale with top axis). The data is gathered during transport of the sample under the Kelvin probe.

Fig. 4 shows the Kelvin probe transient of the $\mathrm{Si}$ sample. The surface voltage shows three constant levels, the first for the Si test sample, the last for the stainless steel foil, with the 19-mm wide Sn tape in-between. The corresponding gradients are shown as well. We identify the changes in sample-probe distance, i.e. the sample topography, with the observed variations in the gradient; first +60 a.u. then -120 a.u. These values correspond to changes in the surface voltage of about $-25 \mathrm{mV}$ and $+50 \mathrm{mV}$, respectively. We conclude that the effect of height is relatively small with respect to the changes in surface voltage due to work function difference.

We apply this knowledge to in-situ roll-to-roll measurements of doped Si layers. The Si layers are deposited directly on the steel carrier foil. On the first $50 \mathrm{~cm}$ of the foil, intrinsic Si was deposited. Then the $\mathrm{PH}_{3}$-flow was increased stepwise during roll-to-roll deposition such that the n-type doping of the layer increased accordingly. The Si coverage ends at 170 $\mathrm{cm}$. Fig. 5 shows the scan of the surface voltage as a function of the position along the foil direction.
First, the work function was measured in roll-toroll mode with a web speed of $5 \mathrm{~cm} / \mathrm{min}$. Data points were taken every $0.88 \mathrm{sec}$, that is every $0.73 \mathrm{~mm}$. The surface voltage was measured without adjusting the gradient. This can cause a certain amount of deviation of the signal over time if the gradient (or probe-surface distance) varies. In a second inspection run, the work function was determined statically at fixed distance intervals. In this run, the gradient was adjusted to 500 a.u. for each measurement. This assures us that the sample-probe distance remains constant, as explained in section 2.2 and Fig. 3.

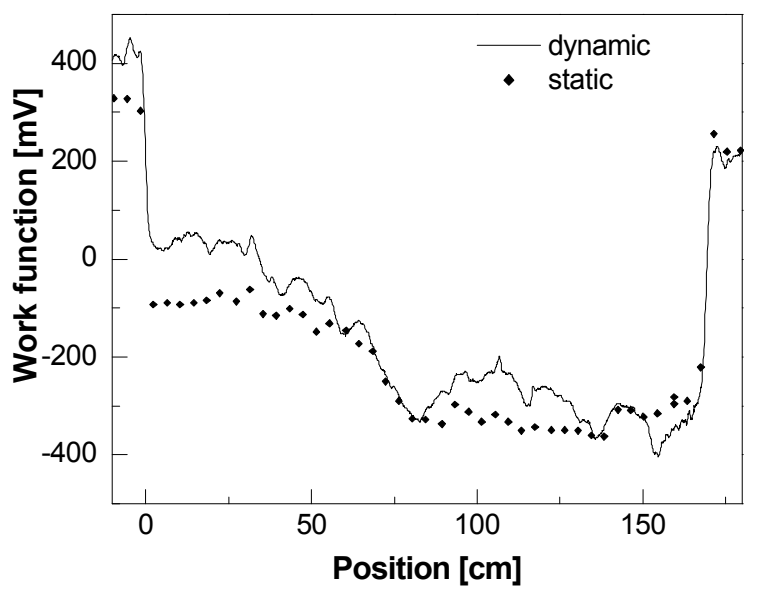

Figure 5 Work function as a function of position on the foil. Initially, the work function is determined continuously ("dynamic" solid line) in roll-to-roll mode. The n-type doping concentration during the deposition of the Si layers is increased linearly from $50 \mathrm{~cm}$ onwards. The second data set "static" was measured at 5-cm distance; during the each measurement, the foil was not moving (see text).

We see that the work function clearly shows the transition at 0 and $170 \mathrm{~cm}$ between steel and (doped) $\mathrm{Si}$. Between 0 and $50 \mathrm{~cm}$ the work function is rather constant, corresponding to the intrinsic layer. Starting at $50 \mathrm{~cm}$, the work function starts to decrease with increasing n-type doping concentration during deposition, as the work function is proportional to the natural logarithm of the donor density. For higher n-type doping concentration flow the work function stabilises, suggesting that the effective donor concentration in the n-type layer no longer increases.

We also see that the dynamic measurements and the static measurements show the same qualitative behaviour in these preliminary scans. Quantitatively, there are some deviations. However, if we plot the gradient during the dynamic measurement as a function of the location, we see the same transient as for the observed difference. The gradient curve shows a linear trend with sinusoidal contribution with a period identical to the circumference of the pick-up roll. Fu- 
ture experiments should improve the in-situ control of the gradient and thereby the accuracy of the dynamic determination of the surface voltage.

Finally, we look whether the Kelvin probe can be used to measure the open-circuit voltage directly, without the need of contacts. The surface photovoltage is determined ex-situ for a range of pin a-Si solar cells on FTO-coated glass. The SPV is plotted against the open-circuit voltage in Fig. 6. The samples were deposited with a variety of layer thicknesses and plasma conditions to create a range of open-circuit voltages. We've divided the samples in two series, because the sample handling and mounting changed significantly at this point of the research. This caused a large shift of the linear relation between the open-circuit voltage and the surface photovoltage.

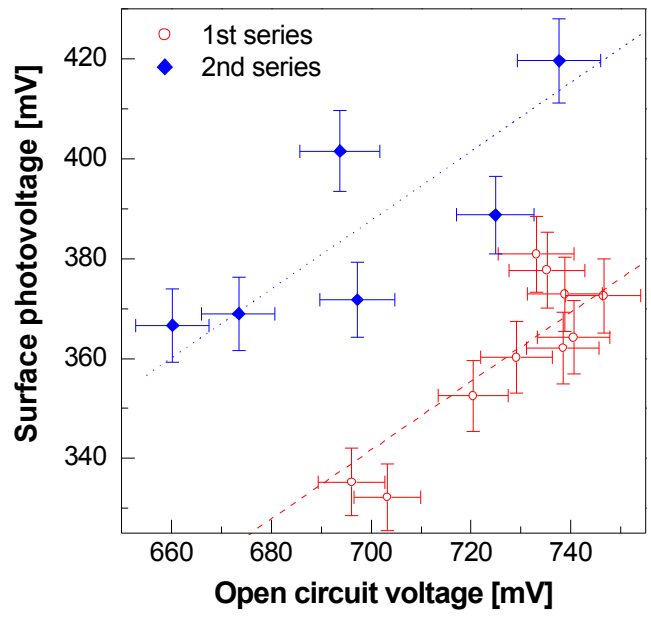

Figure 6 The surface photovoltage as a function of the open-circuit voltage for a-Si pin cells on Asahi glass. The dashed and dotted lines are linear fits to series 1 and 2 with the same slope.

The absolute difference between the $\mathrm{V}_{\mathrm{oc}}$ and the SPV is related to two main differences in the measurement set-up. i) Whereas the $V_{o c}$ is measured with light on the p-side, the SPV is illuminated from the $\mathrm{n}$ side. ii) The $V_{o c}$ is determined with the AM1.5 spectrum; however the intensity of the LED illumination in the SPV is only about a third of that. Furthermore, the spectral distribution of the LED light is quite different from the solar spectrum. This confirms that the KP measurement is very sensitive, both to sample (surface) properties and to environmental variables. Nonetheless, when everything else is constant, we observe a linear correlation between the SPV and the $V_{\text {oc }}$. At the current state of knowledge, we can estimate the $\mathrm{V}_{\mathrm{oc}}$ for an independent substrate with an accuracy of $\pm 25 \mathrm{mV}$. We assume that for measurements along a roll-to-roll deposited solar cell stack, this accuracy will be much better.

\section{Conclusions}

We have shown inline, contactless measurements under deposition conditions using an in-situ Kelvin probe equipped with a surface photovoltage unit. The surface photovoltage of pin a-Si solar cells are linearly related to their open-circuit voltages, when the $\mathrm{KP}$ probe samples are prepared in the same way. Furthermore, the KP can be operated in-situ during roll-roll Si deposition, recording the donor density variation along the foil by way of the work function. To conclude, we have shown that the inline Kelvin probe can be used to continuously monitoring the doped layer quality and the open circuit voltage during roll-to-roll fabrication.

Acknowledgements We'd like to thank Camile Devilee and Patrick Jansen for practical assistance.

\section{References}

[1] M. Izu and T. Ellsion, Sol. En. Mat. Sol. Cells 78, 613 (2003).

[2] A. Takano and T. Kamoshita, Jpn. J. Appl. Phys. 43, 7976 (2004).

[3] J. Löffler et al., in: Proc. 34th IEEE PV Spec. Conf., Philadelphia, USA, 2009.

[4] R.W. Collins, A. S. Ferlauto, G. M. Ferreira, Chi Chen, Joohyun Koh, R. J. Koval, Yeeheng Lee, J. M. Pearce and C. R. Wronski, Sol. En. Mat. Sol. Cells 78, 143 (2003).

[5] A. Hadjadj, P. St'ahel, P. Roca i Cabarrocas, V. Paret, Y. Bounouh and J.C. Martin, J. Appl. Phys. 83, 830 (1998).

[6] G. Harbeke and L. Jastrzebski, J. Electrochem. Soc. 137, 696 (1990).

[7] B.B. van Aken et al., in: MRS Conf. Proc.: Amorphous and polycrystalline thin-film silicon, San Francisco, USA, 2008, pp. 1066.

[8] I.D. Baikie, S. Mackenzie, P.J." . Estrup and J.A. Meyer, Rev. Sci. Instr. 62, 1326 (1991). 